\title{
Signatures of Adaptation in Mitochondrial Genomes of the Palearctic Subterranean Voles (Arvicolinae, Rodentia)
} \author{
Natalia Abramson ${ }^{1}$ \\ 1 Zoological Institute RAS, Saint-Petersburg, 199034, Russia \\ 2 Saint-Petersburg State University, Saint-Petersburg, 199034, Russia \\ * Correspondence: olga.v.bondareva@gmail.com
}

Olga Bondareva ${ }^{*}$, Evgeny Genelt-Yanovskiy ${ }^{1}$, Tatyana Petrova ${ }^{1}$, Semen Bodrov ${ }^{1}$, Antonina Smorkacheva ${ }^{2}$ and

\begin{abstract}
The current study evaluates the selection signals in the evolution of mitochondrial DNA of voles, subfamily Arvicolinae, during the colonization of subterranean environments. The comparative sequence analysis of mitochondrial protein-coding genes of eight subterranean vole species (Prometheomys schaposchnikowi, three species of the genus Ellobius: E. talpinus, E. fuscocapillus and E. lutescens, two species of the genus Terricola: T. subterraneus and T. daghestanicus, Lasiopodomys mandarinus and Hyperacrius fertilis) and their closest aboveground relatives using codon-substitution models was applied. The highest number of selection signatures was detected in genes ATP8 and CYTB. The relaxation of selection was observed in most mtDNA protein-coding genes. In mole voles (genus Ellobius) the signatures of adaptive evolution of mitochondrial genes related to subterranean niche were most pronounced. The number of selection signatures was found to be independent of the evolutionary age of the lineage but fits the degree of specialization to the subterranean niche. The common trends of selective pressures were observed among the evolutionary ancient and highly specialized subterranean rodent families and phylogenetically young lineages of voles. It suggests that the signatures of adaptations in individual mitochondrial protein-coding genes associated with the colonization of the subterranean niche may appear within a rather short evolutionary timespan.
\end{abstract}

Keywords: selective pressures, mitochondrial protein-coding genes, subterranean voles, adaptations, subterranean lifestyle.

\section{Introduction}

Being among the most diverse groups of mammals, rodents colonized major terrestrial habitats all over the world. Most species dwell on the surface, yet many species are able to dig complex tunnels where they nest, rest, and retreat to protect from predators. These forms are commonly classified as fossorial, i.e., they dig burrows and tunnels yet spend only a part of their active time there. Some are nearly completely subterranean they build networks of underground corridors, where most of their activities, including foraging, take place and therefore they are referred as "truly" subterranean [1]. There are approximately 250 species of fossorial and subterranean rodents (combined in 38 genera, six families), that are distributed across all continents except Australia and Antarctica [1].

Subterranean rodents represent a perfect study system for testing hypotheses about adaptive evolution during the colonization of a novel and highly contrasting habitat on the background of aboveground species. They inhabit the environment characterized by high levels of carbon dioxide, low levels of oxygen, and relatively constant temperature and humidity [2].

Voles and lemmings, subfamily Arvicolinae, are the youngest rodent group that emerged via the fastest documented adaptive radiation among recent mammals. The number of extant arvicoline rodents is eight times greater than in the sister subfamily Cricetinae, the most recent common ancestors of both are known in the fossil record from the 
Late Miocene, ca $10 \mathrm{Ma}$ [3]. The earliest arvicolines are known from the Late Miocene both in Eurasia and North America [3,4] and nowadays they colonize almost all landscapes and habitats in the Northern Hemisphere. Arvicolinae provides a unique opportunity for testing various adaptive hypotheses, including these about the evolution towards fossoriality, with a comparative method. The members of this phylogenetically young group display a full spectrum of substrate utilization patterns from surface-dwelling and even arboreal form (e.g. tree voles, Arborimus Taylor, 1915 [5,6] to highly specialized subterranean species (Ellobius Fischer, 1814 and Prometheomys Satunin, 1901 [7]).

Mitochondrial genome historically has been assumed to evolve neutrally [8], and protein-coding genes (e.g. CYTB, COX1) for a long time have been used in phylogenetic reconstructions of various taxonomic groups. Growing number of recent studies, however, provided evidence for non-neutral variation in various parts of the mitogenome. The selective neutrality assumption of the mitochondrial DNA (mtDNA) is simplistic as mitochondrial protein-coding genes are involved in oxidative phosphorylation and responsible for the production of up to $95 \%$ of the energy of eukaryotic cells. Due to the importance of this biochemical pathway, evaluating selective pressures acting on mtDNA proteins could provide key insight into the adaptive evolution of the mtDNA genome as has been suggested by recent empirical evidence $[9,10]$. So, positive selected signals have been detected in various organisms related to shifts in their ecology that imply changes in the metabolic requirements [11].

Following the introduction of computationally tractable codon substitution models $[12,13]$ nearly two decades ago, there has been a persistent interest in using these models to study the past action of natural selection on protein-coding genes. Positive selection can be inferred whenever the estimated ratio $(\omega)$ of non-synonymous $(d N)$ to synonymous $(d S)$ substitution rates significantly exceeds one [14,15]. Using improved natural selection evaluation approaches, state of molecular research on species with altered energy expenditures shows the adaptability of the mitochondrial genome. For instance, birds and bats require a metabolic rate which is 3-5 times higher than the maximum observed during training at ground mammals of similar size [16,17]. Evaluation of selection on mitochondrial genes of bats showed that the number of nonsynonymous substitutions exceeds synonymous in genes ND2, ND3, ND4L, ND4, ND5, ND6, and COX3 and, therefore, the value $\omega$ is significantly higher than in other mammals [18]. Hence, many studies carried out on different species show that the variability of mitochondrial genomes may have adaptive significance [19-24].

Da Silva et al. [25] found a significantly higher $\omega$ in the CYTB gene among independent lineages of subterranean rodents (tuco tucos, coruros, pocket gophers, and mole rats) as compared to their aboveground relatives, suggesting a connection between directional selection of this gene and the niche shift to subterranean. Research on subterranean representatives of two closely related groups Octodontidae and Ctenomyidae: Ctenomys Blainville, 1826 and Spalacopus Wagler, 1832, showed the presence of positive selection in all mitochondrial genes except the ND3 gene. Our previous study [26] also showed that selection pressure is relaxed at the mitochondrial CYTB gene in Arvicolinae subterranean species.

In the current study we were aimed at evaluating the selection signals in mitochondrial genomes of the subterranean Palaearctic voles on the background of their closest aboveground relatives using the comparative sequence analysis. By considering complete mitogenome sequences, we evaluate the magnitude of selective pressures on mitochondrial genes that occurred on the branches leading to subterranean Arvicolinae. Thus, we tested the following hypotheses 1 ) the adaptive signals observed by us previously in the analysis of gene CYTB are also traceable and uniformly distributed among the rest of mitogenome; 2) the selective pressures in mitochondrial genomes of the phylogenetically young subterranean lineages of voles are convergent or have common trends with ancient and highly specialized subterranean rodent families; 3) the detected selective signals related to evolutionary age of the species. 


\section{Materials and Methods}

\subsection{Taxa selection and samples}

Totally we analyzed 27 complete published mitochondrial genomes of Arvicolinae [Abramson et al 2021], sequences of representatives of the sister subfamily Cricetinae were mined from NCBI Genbank [27-40] to be used as an outgroup (Supplementary Table 1). Hereinafter, we use the taxonomic classification following Gromov and Polyakov [41], Musser and Carleton [42], Abramson and Lissovsky [43].

For the purposes of this study, we categorized species as subterranean or aboveground based on their behavioral habits rather than morphological traits. A species was considered subterranean if it is known to conduct the majority of their life underground, perform regular digging activities, and their aboveground excursions are limited to the vicinity of burrow openings [44,45]. Within our dataset, eight species in five genera seem to fit these definitions: Ellobius talpinus Pallas, 1770; Ellobius lutescens Thomas, 1897 and Ellobius fuscocapillus Blyth, 1843; Terrícola subterraneus de Selys-Longshamps, 1836; Terricola daghestanicus Shidlovsky, 1919; Lasiopodomys mandarinus Milne-Edwards, 1871; Hyperacrius fertilis True, 1894 and Prometheomys schaposchnikowi Satunin, 1901.

Two arvicoline genera considered "truly subterranean" are Ellobius Fischer, 1814 (mole voles) and Prometheomys Satunin, 1901 (the long-claw vole) [1,2]. They not only live, forage, and reproduce almost exclusively underground, but also demonstrate a set of obvious morphological and life-history modifications associated with this lifestyle. Both Ellobius and Prometheomys have short soft pelage, small/tiny eyes and reduced ears. Moreover, mole voles have highly modified skull with prominent extrabuccal incisors which are the characteristic of chisel-tooth diggers $[7,46]$ whereas the long-claw vole is a specialized scratch digger [47]. In Terricola Fatio, 1867 voles, the tendency towards fossorial mode of life is thought to result in short dense fur, small eyes, short pinnae hidden in the fur, and the characteristics of slow life history [48-52]. Another fossorial arvicoline, the mandarin vole Lasiopodomys mandarinus does not seem to display marked morphological adaptations that would facilitate digging but living in extended family groups with cooperative tunnel construction [45,53]. The last one, Kashmir vole Hyperacrius fertilis is poorly studied but has been classified as subterranean by Begall et al [1]. Arvicola amphibius Linnaeus, 1758 is often listed among subterranean forms as within this species both semi aquatic and subterranean populations exist. However, the sample we sequenced and included in the analysis is from the semi aquatic population, so we referred it to the background branch.

Subdivision into groups was carried out according to the principle of phylogenetic nearest aboveground taxa selection and more distant as outgroup (Figure 1) for all analysis. Phylogenetic nearest taxa for $H$. fertilis were selected according to its specified phylogenetic position in the tribe Arvicolini [27]. Two different sets were chosen for P. schaposchnikowi, considering its monotypy and basal position within the subfamily Arvicolinae. Thus, we first test against sister to all Arvicolinae hamsters (Group E) from Cricetinae subfamily and second against representatives of the Arvicolinae (Group F) that constitute the so-called first radiation within the subfamily. 

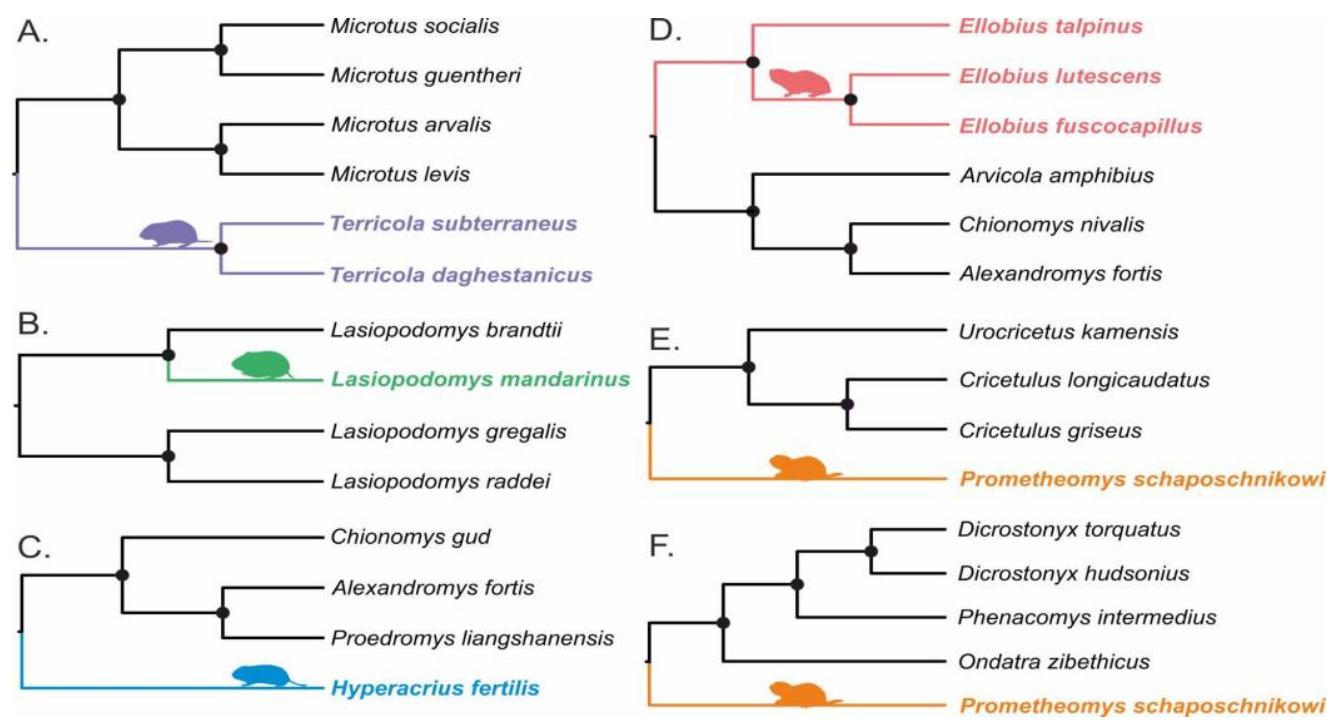

Figure 1. Trees used for tracing signatures of the selection pressures on the subterranean vole species. Fifty-percent majority rule consensus trees from Bayesian inference analysis are given. Subterranean species are shown in color. Black circles show nodes with Bayesian posterior probabilities above 0.99 .

\subsection{Alignment}

The sequences of complete mitochondrial genomes were aligned using the Mauve 1.1.1 [54] implemented as the Geneious Prime 2019.1 plugin (https://www.geneious.com). The concatenated sequence of 13 protein-coding mitochondrial genes was separately aligned using MAFFT 7.222 [55].

\subsection{Phylogenetic reconstructions}

Phylogenetic reconstructions were carried out in the MrBayes 3.2.2 [56] using either seven nuclear genes $(6,421 \mathrm{bp})$ (see methods in [26]) or 13 protein-coding mitochondrial genes $(11,417 \mathrm{bp})$ - in case of $H$. fertilis (since nuclear genes are not available for this species). At this taxonomic level, the topology did not differ depending on nuclear or mitochondrial data on which the phylogenetic reconstruction was built.

The following analysis parameters were set: $\mathrm{nst}=$ mixed and the gamma distribution of the rates of substitutions between sites, the division into partitions by genes was used. Each analysis started with a random tree and had two replicates with four Markov chains (MCMC) and 1 million generations, with the results recorded every hundredth generation. Stationarity and convergence of separate runs were assessed using ESS statistics in Tracer v1.6 [57]. The trees were visualized using the FigTree v1.6 program (http://tree.bio.ed.ac.uk/software/figtree/).

\subsection{Base mitochondrial statistics analysis}

The base nucleotide composition was calculated in Geneious Prime 2019.1 (https://www.geneious.com). The displacement in the nucleotide composition (GC-skew) was studied by calculating the relative frequencies of nucleotides $C$ and $G[58,59]$ using the BioSeqUtils package in BioPython [60] in Python 3.0. The difference in the mean values was checked using the unpaired Wilcoxon test in the R software v.3.4.4 [61]. The mitochondrial genome of $H$. fertilis was excluded from this analysis because of its incompleteness [27] and the possibility of introducing errors into the analysis. 


\subsection{Convergent and parallel amino acids substitution detection}

The evaluation of both convergent and parallel amino acid substitutions was carried out by the ProtParCon program [62] with a further search for amino acids characteristic only for subterranean rodents: we looked for replacements occurring at least in three subterranean taxa using a script on Python 3. The significance of the substitution frequency was estimated with ProtParCon subfunction using additionally Holm multiple adjustments in R software v.3.4.4.

\subsection{Analysis of selective pressures}

Selective pressures were estimated as variation in the levels of non-synonymous $(d N)$ and synonymous $(d S)$ substitutions, as well as $\omega$ using several approaches. We apply three ones: classic codeml program $[63,64]$ with the comparisons between free-branch \& M0 and free-branch \& neutral-branch models; improved branch-site model - aBSREL (an adaptive branch-site REL test for episodic diversification [65]) and RELAX [66] to test whether the strength of natural selection has been relaxed or intensified along a specified set of branches. Also, we search sites under positive selection with MEME (Mixed Effects Model of Evolution, [67]). All programs except codeml are implemented in the DataMonkey webserver (datamonkey.org, [68,69]).

We used a full set of mitochondrial protein-coding genes $(n=13)$ for each subterranean rodent. For branch-site analysis the total set of species was subdivided into several subsets so that each subset contained a subterranean species and its phylogenetically closest or sister aboveground species (Figure 1). The estimation for P. schaposchnikowi was assessed twice, in respect to hamsters (Figure 1, E) and to other representatives of the Arvicolinae (Figure 1, F) due to its basal position within the subfamily. The calculated pvalues of all likelihood-ratio tests (LRTs) were corrected using Holm multiple adjustments in R software v.3.4.4.

The classical codeml program [63,64] was used with the ete-toolkit interface [70]. A branch models assume significant differences between $\omega$ values for marked branches (foreground or $\omega \mathrm{frg}$ ) and the rest branches of the tree (background branches or $\omega \mathrm{bkg}$ ). The branch model-based approach allows estimation of selection level at an individual subterranean species (colored on Figure 1) compared only with phylogenetically close aboveground taxa (marked black on Figure 1). In all the analyses, subterranean species were marked as foreground and aboveground taxa as background branches. For each subterranean species (or group of species for Ellobius and Terricola) analysis was implemented with free-branch model (b_free, where $\omega$ frg and $\omega \mathrm{bkg}$ are free), neutral-branch model (b_neut, where $\omega \mathrm{frg}$ is fixed to one) and M0 model, where all branches evolve at the same rate. LRT was calculated to compare different models. The comparison between freebranch and M0 shows whether foreground branches have $\omega$ significantly different from the rest of the tree. And the comparison between free-branch and neutral-branch models detects if the value of $\omega$ frg was significantly higher than 1 . The values 999 and 0,001 were regarded as errors.

The aBSREL differs from other branch-site model implementations by inferring the optimal number of $\omega$ classes for each branch. After aBSREL fits the full adaptive model, the LRT is performed at each branch and compares the full model to a null model where branches are not allowed to have rate classes of $\omega>1$. RELAX was employed to test changes in the intensity of selection (relaxation or intensification) in phylogenetic branches. A significant $\mathrm{K}>1$ estimate indicates that selection strength has been intensified along the test branches, while a significant $\mathrm{K}<1$ indicates that selection strength has been relaxed along the test branches. MEME employs a mixed-effects maximum likelihood approach to test the hypothesis that individual sites have been subjected to episodic positive 
or diversifying selection. So, MEME aims to detect sites evolving under positive selection under a proportion of branches. We changed the confidence level manually from 0.1 to 0.05 for more significant results.

\section{Results}

\subsection{Comparison of base nucleotide composition and gene order}

Variation in GC-content among mitogenomes of subterranean species was lower than among aboveground species (Figure 2). There were no differences in mean values of GC-content and GC-skew. Gene order among all studied mitochondrial genomes remained unchanged.

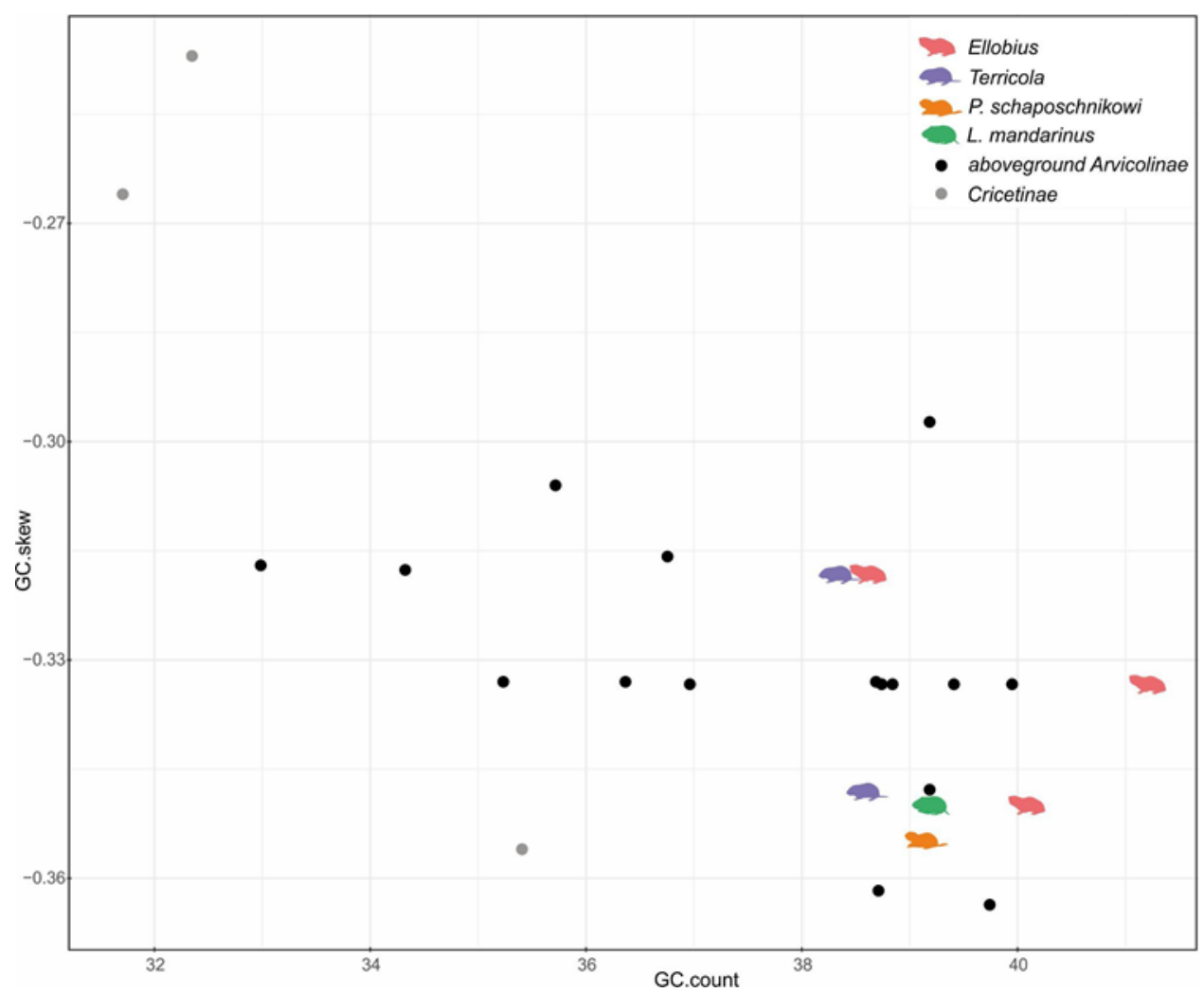

Figure 2. Variation of GC-content and GC-skew values in mitochondrial genomes of Arvicolinae. Black dots - aboveground vole species (Arvicolinae), grey dots - hamsters (Cricetinae), color symbols - subterranean vole species.

\subsection{Selection relaxation or intensification}

Significant selection signs were detected in three subterranean taxa using ete-evol workflow based on codeml (Table 1, for all data see Table S2). According to this analysis, eight mitochondrial protein-coding genes in Ellobius show significantly higher $d N / d S$ values compared to the background taxa of Arvicola amphibius, Chionomys nivalis Martins, 1842 and Alexandromys fortis Büchner, 1889. Two genes (COX3 and CYTB) were detected as genes with higher $\omega$ in L. mandarinus compared to other species of genus Lasiopodomys. Only COX3 demonstrated signatures of selection in P. schaposchnikowi on the background of Ondatra zibethicus Linnaeus, 1766, true and collared lemmings (Table 1). Higher $d N / d S$ values observed in foreground branches in all above mentioned comparison pairs indicated signatures of relaxed selection in mitochondrial protein-coding genes of subterranean rodents (Table 1, Table S2). 
All genes in Ellobius except ATP8 showed signatures of non-neutral selection together with all 13 mitochondrial protein-coding genes of two Terricola species and seven genes of L. mandarinus (Table S2).

Table 1. Estimation of $\omega$ in ete-toolkit using a branch model. Fg - foreground branch (subterranean species), Bg - background branch (aboveground species). Subterranean species indicated by color on Figure 1. LRT - likelihood ratio test p-value for models' comparison, M0 - one-ratio model, b_fee - free-branch model. Only significant results are given in this table, all values can be found in Table S2.

\begin{tabular}{|c|c|c|c|}
\hline Gene & $\mathrm{Fg}, \omega 1$ & $\mathrm{Bg}, \omega 0$ & $\begin{array}{l}\text { Adjusted p-values } \\
\text { LRT b_free \& M0 }\end{array}$ \\
\hline \multicolumn{4}{|c|}{ Ellobius \& group D } \\
\hline ATP6 & 0,13 & 0,04 & 3.43E-04 \\
\hline ATP8 & 0,74 & 0,16 & 0,0052 \\
\hline COX1 & 0,03 & 0,01 & $5.04 \mathrm{E}-05$ \\
\hline COX2 & 0,22 & 0,02 & 1.17E-09 \\
\hline CYTB & 0,06 & 0,02 & $3.74 \mathrm{E}-04$ \\
\hline ND1 & 0,06 & 0,03 & 0,0125 \\
\hline ND4 & 0,09 & 0,04 & 0,0017 \\
\hline$N D 4 L$ & 0,18 & 0,04 & 0,0051 \\
\hline \multicolumn{4}{|c|}{ P. schaposchnikowi \& Group E } \\
\hline COX3 & 0,05 & 0,01 & 0,0136 \\
\hline \multicolumn{4}{|c|}{ L. mandarinus \& Group B } \\
\hline COX3 & 0,12 & 0,03 & 0,0324 \\
\hline CYTB & 0,23 & 0,03 & $1.70 \mathrm{E}-04$ \\
\hline
\end{tabular}

The aBSREL approach also found evidence of episodic positive selection in analyzed subterranean rodents (Figure 1). It is observed in COX2 for E. lutescens (but not in other species of the genus Ellobius) and in two genes for P. schaposchnikowi: ATP8 as compared with Arvicolinae species (Group F) and ND5 in respect to hamsters (Group E) (Table 2).

Table 2. Estimation of $\omega$ using aBSREL. Name - branch of interest, B - optimized branch length, LRT - likelihood ratio test, Test pvalue - p-value corrected for multiple testing.

\begin{tabular}{|l|l|l|l|l|l|l|}
\hline Group & Gene & Name & B & LRT & Test p-value & $\boldsymbol{\omega}$ distribution over sites \\
\hline $\begin{array}{l}\text { Ellobius \& Group D } \\
\text { COX2 }\end{array}$ & E. lutescens & 0.0072 & 17.9838 & 0.0001 & $\begin{array}{l}\omega 1=0.238(81 \%) \\
\omega 2=15.4(19 \%)\end{array}$ \\
\hline $\begin{array}{l}\text { P. schaposchnikowi \& } \\
\text { Group E }\end{array}$ & ND5 & P. schaposchnikowi & 0.0149 & 4.4686 & 0.0390 & $\begin{array}{l}\omega 1=0.395(89 \%) \\
\omega 2=16.7(11 \%)\end{array}$ \\
\hline $\begin{array}{l}\text { P. schaposchnikowi \& } \\
\text { Group F }\end{array}$ & ATP8 & P. schaposchnikowi & 0.1443 & 6.0817 & 0.0171 & $\begin{array}{l}\omega 1=0.0553(90 \%) \\
\omega 2=\infty(10 \%)\end{array}$ \\
\hline
\end{tabular}


The RELAX analysis showed changes in the intensity of selection in mitochondrial protein-coding genes of three subterranean species compared with aboveground ones (Table 3, Table S3). Eight genes with significant K-values $<1$ and traces of selection relaxation were detected in Ellobius: ATP6, COX1, COX3, CYTB, ND1, ND2, ND3 and ND4. Five genes (COX1, COX3, ND2, ND4, and ND5) were under relaxed selection in P. schaposchnikowi compared to Group F (Figure 1). Selective pressure was also significantly weakened in the COX3 gene of $P$. schaposchnikowi compared to Cricetulus species (Group E). Analysis indicated three genes with signatures of relaxed selection in L. mandarinus: COX1, COX3, and CYTB (Table 3). No genes with a significant relaxation or intensification of selection were found for the remaining subterranean voles: H. fertilis and both investigated Terricola species.

Table 3. Estimation of natural selection intensity using the RELAX approach. LRT - likelihood ratio test, P - adjusted p-value, K selection intensity parameter. Only significant results are given in this table, all values can be found in Table S3.

\begin{tabular}{|c|c|c|c|}
\hline Gene & LRT & $\mathbf{P}$ & $\mathbf{K}$ \\
\hline \multicolumn{4}{|c|}{ Ellobius \& group D } \\
\hline ATP6 & 38,9000 & 5.785E-09 & 0,1700 \\
\hline COX1 & 24,8820 & 7.308E-06 & 0,4070 \\
\hline COX3 & 9,0750 & 0,0175 & 0,1320 \\
\hline CYTB & 17,4720 & 0,0003 & 0,4816 \\
\hline ND1 & 14,7850 & 0,0011 & 0,6414 \\
\hline ND2 & 23,7070 & $1.232 \mathrm{E}-05$ & 0,3624 \\
\hline ND3 & 7,977 & 0,0282 & 0,5980 \\
\hline ND4 & 14,1950 & 0,0013 & 0,1628 \\
\hline \multicolumn{4}{|c|}{ P. schaposchnikowi \& Group F } \\
\hline COX1 & 18,8005 & 0,0002 & 0,3875 \\
\hline COX3 & 12,4548 & 0,0042 & 0,4027 \\
\hline ND2 & 21,2713 & $4.788 \mathrm{E}-05$ & 0,1834 \\
\hline ND4 & 30,1175 & $5.291 \mathrm{E}-07$ & 0,5331 \\
\hline ND5 & 9,9163 & 0,0147 & 0,3350 \\
\hline \multicolumn{4}{|c|}{ P. schaposchnikowi \& Group E } \\
\hline COX3 & 18,5788 & 0,0002 & 0,3382 \\
\hline \multicolumn{4}{|c|}{ L. mandarinus \& Group B } \\
\hline COX1 & 8,8720 & 0,0319 & 0,4922 \\
\hline COX3 & 11,1457 & 0,0101 & 0,0071 \\
\hline СУТВ & 22,5555 & 2.652E-05 & 0,3003 \\
\hline
\end{tabular}


A number of orthologous genes showed relaxation of selection in several subterranean species. For instance, COX1 and COX3 genes demonstrated relaxation in all three Ellobius species, P. schaposchnikowi and L. mandarinus. Some genes were detected under relaxed selection in Ellobius species and P. schaposchnikowi (ND2, ND4) or Ellobius species and L. mandarinus (CYTB).

\subsection{Estimation of selective pressures in sites of protein-coding genes}

We applied the MEME approach for each subterranean taxon individually (Figure 1) to search for sites under positive selection in all mitochondrial protein-coding genes. Results of this analysis are shown in Table 4.

Table 4. Sites under positive selection found in mitochondrial genes using the MEME approach. N/A - not analyzed, no sites analysis did not show sites under positive selection. Homologous site found for several species in COX1 indicated by bold.

\begin{tabular}{|c|c|c|c|c|c|c|}
\hline Gene & Ellobius & $\begin{array}{l}\text { P. schaposchnikowi \& } \\
\text { Group F }\end{array}$ & $\begin{array}{l}\text { P. schaposchnikowi } \\
\text { \& Group E }\end{array}$ & $\begin{array}{l}\text { L. } \\
\text { mandarinus }\end{array}$ & H. fertilis & Terricola \\
\hline ATP6 & no sites & no sites & no sites & 76 & no sites & no sites \\
\hline ATP8 & 64 & 22,41 & no sites & 60 & 53,63 & no sites \\
\hline COX1 & 434 & no sites & 434,484 & no sites & no sites & no sites \\
\hline COX2 & no sites & no sites & no sites & 218 & no sites & no sites \\
\hline COX3 & no sites & no sites & no sites & no sites & no sites & no sites \\
\hline СУTB & no sites & 296 & 380 & $\begin{array}{l}23,57,67,226, \\
329,371\end{array}$ & 82 & no sites \\
\hline ND1 & no sites & no sites & no sites & 65,299 & no sites & no sites \\
\hline ND2 & 69 & $83,199,281,320$ & no sites & 235 & N/A & no sites \\
\hline ND3 & no sites & no sites & no sites & 20 & no sites & no sites \\
\hline ND4 & $\begin{array}{l}22,169,189, \\
256\end{array}$ & 188 & $\begin{array}{l}19,25,107,171,188 \\
205,351\end{array}$ & $155,350,441$ & no sites & no sites \\
\hline$N D 4 L$ & 17 & no sites & no sites & no sites & no sites & no sites \\
\hline ND5 & 36 & $59,441,491,511$ & $213,542,551,602,603$ & 311,364 & N/A & no sites \\
\hline ND6 & 111 & no sites & 4,81 & no sites & no sites & no sites \\
\hline
\end{tabular}


Various sites under positive selection have been found in the orthologous mitochondrial genes. Its amount differs in several gene groups: so, genes CYTB, ND4 and ND5 turned out to be the richest ones while no sites at all were detected in the COX3 gene. The largest number of sites under positive selection were found for P. schaposchnikowi, Ellobius group and L. mandarinus. In genes ATP6, COX2, ND1 and ND3 we found sites only for $L$. mandarinus and in gene ND4L only for the Ellobius group. Three sites in two genes (ATP8 and $C Y T B$ ) were detected for $H$. fertilis. Above all, homologous site 434 in COX1 gene was detected as being under positive selection both for the Ellobius species and P. schaposchnikowi. No sites under positive selection were traced for two Terricola species.

\subsection{Search for parallel and convergent amino acid substitutions}

Using ProtParCon, we found six amino acid substitutions meeting our search criteria: COX1 Met73Ile, COX3 Ile121Val, ND5 Phe446Leu, CYTB Thr56Ser, CYTB Ile338Val, CYTB Ala357Thr (Table S4), However, all of them appeared to be insignificant upon further verification.

\section{Discussion}

Signatures of selection were recently found in several mitochondrial genes of subterranean rodents $[11,25,71,72]$ using the phylogenetic framework at the family level and over a vast evolutionary period. Here, we applied a similar approach in search for selection traces at a significantly smaller evolutionary timescale and within a lower taxonomic level: several subterranean species of the Arvicolinae subfamily. Comparing different genera within subfamily and different species within one genus that independently switch to the subterranean lifestyle, we aimed to determine whether this fast transition left signatures of selection in mitochondrial protein-coding genes and whether the substitutions occur at homologous or different sites and genes.

Applying various approaches in searching selection signatures in Arvicolinae mitochondrial protein-coding genes, we have found 1) genes with signs of selection relaxation in subterranean species; 2) among subterranean species the number of genes with selection signatures substantially differs.

Gene COX3 demonstrated higher $\omega$ values in codeml branch analysis for both $P$. schaposchnikowi and L. mandarinus and CYTB for L. mandarinus and Ellobius species related to sister aboveground taxa. According to the RELAX analysis, COX1, COX3, ND2 and ND4 show selection relaxation for at least two subterranean species (Figure 3). List of common genes with sites under positive selection discovered in branch-site MEME analysis includes more than half of mitochondrial protein-coding genes: ATP8, COX1, CYTB, ND2, ND4, ND5 and ND6. Genes COX1 and COX3 were found to be the most affected by selection pressure according to the results of several approaches based on complete gene sequence analysis, i.e. excluding MEME results (Figure 3).

\begin{tabular}{|c|c|c|c|c|c|c|c|c|c|c|c|c|c|}
\hline & ND1 & ND2 & $\operatorname{cox} 1$ & $\operatorname{cox} 2$ & $\mathrm{cox} 3$ & ATP8 & ATP6 & ND3 & ND4L & ND4 & ND5 & ND6 & CYTB \\
\hline$E$. & $\mathrm{Cr}$ & $r \mathrm{~m}$ & $\mathrm{crm}$ & ca & $r$ & $\mathrm{~cm}$ & & $\therefore$ & $\mathrm{cm}$ & $\mathrm{crm}$ & $m$ & $m$ & crm \\
\hline P. s. & - & $\mathrm{rm}$ & $\mathrm{rm}$ & - & $\mathrm{cr}$ & a m & - & - & - & $r m$ & arm & $\mathrm{m}$ & $\mathrm{m}$ \\
\hline L & $\mathrm{m}$ & & & $n$ & & & & & - & $\mathrm{m}$ & $\mathrm{m}$ & - & $\mathrm{crm}$ \\
\hline H. f. & - & - & - & - & - & $m$ & - & - & - & - & - & - & $m$ \\
\hline$T$. & - & - & - & - & - & - & - & - & - & - & - & - & - \\
\hline
\end{tabular}

Figure 3. Genes with significant changes in selective pressures for each of the subterranean species. Letters inside each silhouette indicate the analyzes in which a significant result was obtained. E - Ellobius species, P.s. - P. schaposchnikowi, L.m. - L. mandarinus, H.f. - H. fertilis, T. - Terricola species. 
Similar results were obtained by Da Silva et al. [25], who found a significant difference for $\omega$ values in CYTB sequences of subterranean rodents from families Ctenomyidae, Geomyidae and Bathyergidae compared to their aboveground closely related species. Significantly higher $\omega$ ratio in subterranean groups relative to aboveground ones was shown also by [11]. Moreover, the distribution of non-synonymous mutations indicated the considerable changes in $C Y T B$ of animals, which were confronted with more severe hypoxia because of higher altitude and a colder, drier climate and were facing a higher purifying selection pressure [73]. Finally, Tomasco and Lessa [71] found increased values of $\omega$ in COX2 of subterranean coruros Spalacopus (almost 30X) and tuco-tucos Ctenomys (11X) relative to aboveground octodontoids. Our previous studies on the Arvicolinae subfamily also showed the relaxation of selection strength in CYTB reflecting an increased ratio $(\omega)$ in subterranean lineages compared to non-subterranean [26]. Later, indicated changes were demonstrated for all mitochondrial protein-coding genes. Subterranean species south American tuco-tucos and the related coruro in the work of Tomasco and Lessa [71] showed a significantly higher $\omega$ compared to their aboveground counterparts in 11 of 13 mitochondrial genes. Convergent changes were also found between the studied subterranean genera and other mammals adapted to hypoxia. All genes except ND3 show amino acid properties consistent with positive destabilizing selection. Proteins varied in the fraction of strong positively selected amino acid properties per site, from 0.03 in COX3 to 0.38 in ATP8. In most genes one or several (for ND4L, ND5 and COX1) positively selected sites were detected. Tavares and Seuánez [72] findings showed significant selection relaxation in most mitochondrial protein-coding genes of subterranean hystricomorphs (African mole-rats, tuco-tucos, and coruro). Conversely, selection intensification was found to have occurred in three genes in fossorial sciuromorphs (ground squirrels, chipmunks, marmot). Thus, summing up this brief comparison with other studies of selective pressures in mitochondrial genomes of subterranean rodents, we observe the common trends in evolutionary old and highly specialized subterranean rodent families and phylogenetically young lineages of voles.

According to some assumptions [2], such an increase in the level of selection in mitochondrial protein-coding genes could be a consequence of the low effective population size of subterranean rodents. When $\omega$ values are smaller than 1 , the study of a single gene does not allow us to reject alternative, non-selectionist explanations of rate variation, such as a variation in metabolic rate, body mass, population size, and generation time among lineages [74-76]. However, under a relaxation of purifying selection model, the same pattern of variation among lineages is expected in all genes. In contrast, our study found (a) most, but not all genes have greater $\omega$ in subterranean lineages; and (b) different combinations of genes with selection signatures are detected by used approaches in studied species.

We found that some genes with an increased number of selection signatures appear in various analyses more frequently than others. It may be supposed that the number of selection signatures is partly positively correlated with the rate of gene evolution. Mutation rates between mitochondrial gene families are distributed as follows: $A T P>N D>$ CYTB > COX according to Lopez [77]. In accordance with this assumption our results showed that both ATP genes display an increase in signatures of selection in subterranean species, except $T$. subterraneus and $T$. daghestanicus. Moreover, selective traces in genes from the ATP-group are detected in several analyzes: RELAX (ATP6 for genus Ellobius) and aBSREL (ATP8 for P. schaposchnikowi). ATP8 demonstrated positively selected sites in almost all analyzed species, except Terricola species and thus it is one of the two genes in our dataset with most pronounced selection signs (Figure 3). Genes of the ND group vary $\mathrm{r}$ in the degree of selective pressures. Genes ND2, ND4 and ND5 were detected in all of the performed analyzes in three out of five analyzed subterranean species. Aside from positively selected sites detected by MEME, significant changes in selective pressure were found in ND1, ND2, ND4, ND4L and ND5 genes (Figure 3). The CYTB gene shows a significant difference in $\omega$ levels between subterranean and aboveground species and confirmed selection relaxation for Ellobius species and L. mandarinus. These results reproduce 
our earlier findings performed in the analysis of the CYTB alone and with a different set of compared species [26]. In addition to previously reported, we have now detected positively selected sites for P. schaposchnikowi, Ellobius species, L. mandarinus and H. fertilis. Thus, CYTB on a par with ATP8 appears as the gene with maximum selection signatures (Figure 3). An unexpected result was obtained for the COX genes: they show adaptive signatures across all types of analyzes at the same level as more variable $N D$ genes despite the greatest conservatism.

It is remarkable that the largest number of identified genes under selection in our study was found in P. schaposchnikowi, Ellobius species and L. mandarinus (Figure 3). P. schaposchnikowi, the oldest subterranean lineage within the subfamily, represents the first wave of species radiation within Arvicolinae [78] and the earliest split within subfamily $[79,80]$. Ellobius species (tribe Ellobiusini) and L. mandarinus (tribe Arvicolini) are significantly younger and both represent the latest most diverse radiation wave of the Arvicolinae subfamily. Thus, there is no correlation between the number of revealed genes with selection signatures and the evolutionary age of the lineage. For instance, lineages with a similar evolutionary age, as the Ellobius species - H. fertilis - dramatically differ in selective pressures in mitochondrial genes. While for Ellobius species we observe signatures of selection in almost all mitochondrial protein-coding genes, in $\mathrm{H}$. fertilis we found only three sites under positive selection in two genes. The same phenomenon is observed while comparing similar in age subterranean species of the Arvicolini tribe: T. subterraneus, T. daghestanicus and L. mandarinus. While Terricola species do not show any signatures of selection, L. mandarinus in contrast is the second one after the mole voles in the number of such signatures in mitochondrial genes.

In total, the highest number of selection signatures related to the subterranean lifestyle we have found in the Ellobius species which are younger than the oldest representative of the subfamily - subterranean P. schaposchnikowi, by ca. $2 \mathrm{Ma}$. However, if viewed in light of the degree of specialization to the subterranean lifestyle the data obtained seem to be quite logical. The most stressful challenges faced by subterranean rodents are hypoxic/hypercapnic conditions and overheating in the closed burrow system [2]. While both mole voles and long claw voles are highly specialized diggers, they occupy different habitats, and their burrows characteristics as well as foraging methods are quite distinctive. The typical habitats of Ellobius are arid or semi-arid landscapes like steppes, deserts and grasslands. These rodents populate various soil types, including the compact soils of clay deserts, and create rather stable systems of narrow tunnels to forage underground with geophytes $[7,41,46]$. Thus, mole voles should cope with issues which other truly subterranean mammals encounter [2]. The latter in its turn may lead to the changes we observed in the mitochondrial protein-coding genes. To the contrary, P. schaposchnikowi occurs in Caucasian subalpine tall-grass meadows at $1500-2500 \mathrm{~m}$ a.s.1. [51,81,82]. Its burrows are excavated in loose humid soils filled with stones which should increase gas diffusion. Importantly, the superficial foraging tunnels of these voles are unusual in that they seem to be oversized being almost twice as wide as you would expect for a rodent of this size ([82]; personal observations of AVS). This extra space may prevent overheating, hypoxia and hypercapnia. Further, the long-clawed mole-vole is strictly herbivorous, at least in summer. During the foraging bouts, the vole sticks out of the hole, picks up the plants and drags them into the burrow to feed in safety ([83,84]; personal observations of AVS). Therefore, even being limited in foraging activity to the vicinity of burrow openings, long claw voles actually spend a lot of time out of tunnels. Due to the cold climate, burrow architecture and feeding behavior, Prometheomys may avoid some of the physiological challenges which most subterranean species have to cope with.

We found significant differences in selective pressures on mitochondrial protein-coding genes in Lasiopodomys mandarinus and Terricola species despite the similar evolutionary age of these taxa. This difference also may reflect unequal levels of energetic and hypoxic stress resulting from specific characteristics of foraging strategies and burrow architecture. Mandarin voles feed either on geophytes underground or green parts of plants in the immediate vicinity of the burrow entrance. Foraging tunnels of this species are located 
at depths of $10-30 \mathrm{~cm}[85,86]$, and the direct measurements of gas concentrations, temperature and humidity confirmed that animals in the burrow should face hypoxia and hypercapnia. European pine voles inhabit various vegetation communities from broad-leaf forests (T. subterraneus) to alpine meadows (T. daghestanicus) [87]. Similarly to Ellobius, Prometheomys and L. mandarinus, they use complex network of underground runways, and exhibit some external traits associated with fossoriality, and thus are usually referred as subterranean $[52,87]$. However, the slender physique and nimble habits of T. subterraneus (Abramson and Smorkatcheva, unpublished) as well as the characteristics of its tunnel system distinguish this vole from the specialized subterranean rodents. Feeding runways of this species are located in the very surface soil levels (within 5-10 cm) or even just under leaf litter [52]. These runways afford the voles protection from unfavorable weather and predators which explain the tendency of Terricola species for slow life history [88-90], but their depth is probably too shallow to significantly prevent gas diffusion resulting in hypoxic/hypercapnic conditions inside. Unfortunately, almost nothing is known about the ecology of $T$. daghestanicus which inhabits Caucasian alpine steppes and meadows. The fact that the voles of this species are reported to find shelter among rocks [51] suggests that they are not so strictly subterranean. Our results confirm this fact, so we didn't find any changes of selective pressures in mitochondrial genes for this species characteristic for other subterranean species. Unfortunately, we do not have enough data about the $H$. fertilis digging feature and practice to associate it with detected substitutions.

Taken collectively, our results are consistent with a hypothesis that the colonization of the subterranean niche creates a selective regime of positive, directional selection in protein-coding mtDNA. Our data confirms the recent findings obtained by us in the analysis of CYTB sequences and several studies based on subterranean rodent families mitochondrial genomes [25,72]. We indicate the signatures of positive selection in the evolution of mitochondrial DNA in Arvicolinae during colonization of subterranean environments. We observe relaxation of selection in most mtDNA protein-coding genes using $d N / d S$ calculation with branch and branch-site models. Herewith different mitochondrial genes demonstrated various levels of evolutionary changes, so ATP8 and CYTB showed utmost changes in analyzed species. We also observe a discrepancy between the number of detected genes with signatures of selection and evolutionary age of the lineage. The highest number of selection signatures we found in the Ellobius species, not the most ancient among the studied taxa but the most specialized subterranean form. It suggests that the signatures of adaptations in individual mitochondrial protein-coding genes associated with the entering of the subterranean niche may appear within a rather short evolutionary timespan.

\section{Conclusions}

We indicate the signatures of positive selection in the evolution of mitochondrial DNA in Arvicolinae during colonization of the subterranean environment. We found that the number of selection signatures in mitochondrial genes is independent of the evolutionary age of the lineage but positively correlated with the degree of specialization to the subterranean niche. The data obtained suggests that the signatures of adaptations in individual mitochondrial protein-coding genes associated with the entering of the subterranean niche may appear within a rather short evolutionary timespan.

Supplementary Materials: The following are available online at www.mdpi.com/xxx/s1, Table S1: Species and GenBank accession numbers, Table S2: Estimation of $\omega$ in ete-toolkit using a branch model, Table S3: Estimation of natural selection intensity using the RELAX approach, Table S4: Identified parallel substitutions in mitochondrial protein-coding genes.

Author Contributions: OB: Methodology, Software, Investigation, Formal analysis, Data curation, Writing - original draft, Writing - review \& editing, EGY: Methodology, Software, Visualization, Writing - review \& editing, TP: Data curation, Formal analysis and Visualization, SB: Data curation, Writing - review \& editing, AS: Data curation, Writing - original draft, NA: Conceptualization, 
Data curation, Methodology, Resources, Writing - review \& editing, Project administration, Funding acquisition. All authors read and approved the final manuscript.

Funding: This work was supported by the Russian Science Foundation N 19-74-20110 (for OB, SB, EGY, TP and NA) and Russian Foundation for Basic Research (RFBR) N 19-04-00538a (for AS).

Institutional Review Board Statement: Not applicable.

Data Availability Statement: All analyzed mitochondrial genomes available in GenBank, accession numbers given in Table S1.

Acknowledgments: We are grateful to colleagues who shared the material for the study and Dmitry Rasskazov for help with server management.

Conflicts of Interest: The authors declare no conflict of interest.

\section{References}

1. Begall, S.; Burda, H.; Schleich, C.E.; Begall, S. Subterranean Rodents: News from Underground; Springer: Berlin London, 2007.

2. Lacey, E.A., Patton, J.L., Cameron, G.N. Life Underground: The Biology of Subterranean Rodents; University of Chicago Press: Chicago, 2000.

3. Fejfar, O.; Heinrich, W.-D.; Kordos, L.; Maul, L.C. Microtoid Cricetids and the Early History of Arvicolids (Mammalia, Rodentia). Palaeontol Electron 2011, 14, 27A.

4. Martin, R.A. Biochronology of Latest Miocene through Pleistocene Arvicolid Rodents from the Central Great Plains of North America. Coloquios de Paleontologia 2003, 373-383.

5. Swingle, J.K.; Foreman, E.D. Home Range Areas and Activity Patterns of Red Tree Voles ( Arborimus Longicaudus ) in Western Oregon. Northwest Sci 2009, 83, 273-286.

6. Corn, P.S.; Bury, R.B. Distribution of the Voles Arborimus Longicaudus and Phenacomys Intermedius in the Central Oregon Cascades. J Mammal 1988, 69, 427-429.

7. Ognev, S.I. Zveri SSSR I Prilezhashhih Stran (The Mammals of the USSR and Adjacent Countries). Vol VII. Gryzuny (Rodentia).; Publ. Acad. Nauk USSR: Leningrad, 1950; Vol. 7.

8. Avise, J.C. Mitochondrial DNA and the Evolutionary Genetics of Higher Animals. Philos Trans R Soc B 1986, 312, $325-342$.

9. Ruiz-Pesini, E.; Mishmar, D.; Brandon, M.; Procaccio, V.; Wallace, D.C. Effects of Purifying and Adaptive Selection on Regional Variation in Human MtDNA. Science 2004, 303, 223-226.

10. Bazin, E.; Glémin, S.; Galtier, N. Population Size Does Not Influence Mitochondrial Genetic Diversity in Animals. Science 2006, $312,570-572$.

11. Tomasco, I.H.; Lessa, E.P. Two Mitochondrial Genes under Episodic Positive Selection in Subterranean Octodontoid Rodents. Gene 2014, 534, 371-378.

12. Goldman, N.; Yang, Z. A Codon-Based Model of Nucleotide Substitution for Protein-Coding DNA Sequences. Mol Biol Evol 1994, 11, 725-736.

13. Muse, S.V.; Gaut, B.S. A Likelihood Approach for Comparing Synonymous and Nonsynonymous Nucleotide Substitution Rates, with Application to the Chloroplast Genome. Mol Biol Evol 1994, 11, 715-724.

14. Anisimova, M.; Kosiol, C. Investigating Protein-Coding Sequence Evolution with Probabilistic Codon Substitution Models. Mol Biol Evol 2009, 26, 255-271.

15. Delport, W.; Scheffler, K.; Seoighe, C. Models of Coding Sequence Evolution. Brief Bioinform 2009, 10, 97-109.

16. Thomas, S.P.; Suthers, R.A. The Physiology and Energetics of Bat Flight. J Exp Biol 1972, 57, 317-335.

17. Maina, J.N. What It Takes to Fly: The Structural and Functional Respiratory Refinements in Birds and Bats. J Exp Biol 2000, 203, 3045-3064.

18. Shen, Y.-Y.; Liang, L.; Zhu, Z.-H.; Zhou, W.-P.; Irwin, D.M.; Zhang, Y.-P. Adaptive Evolution of Energy Metabolism Genes and the Origin of Flight in Bats. Proc Natl Acad Sci USA 2010, 107, 8666-8671. 
19. Hassanin, A.; Ropiquet, A.; Couloux, A.; Cruaud, C. Evolution of the Mitochondrial Genome in Mammals Living at High Altitude: New Insights from a Study of the Tribe Caprini (Bovidae, Antilopinae). J Mol Evol 2009, 68, $293-310$.

20. Luo, Y.; Yang, X.; Gao, Y. Mitochondrial DNA Response to High Altitude: A New Perspective on High-Altitude Adaptation. Mitochondrial DNA 2013, 24, 313-319.

21. Wang, Y.; Shen, Y.; Feng, C.; Zhao, K.; Song, Z.; Zhang, Y.; Yang, L.; He, S. Mitogenomic Perspectives on the Origin of Tibetan Loaches and Their Adaptation to High Altitude. Sci Rep 2016, 6, 29690.

22. Yu, L.; Wang, X.; Ting, N.; Zhang, Y. Mitogenomic Analysis of Chinese Snub-Nosed Monkeys: Evidence of Positive Selection in NADH Dehydrogenase Genes in High-Altitude Adaptation. Mitochondrion 2011, 11, 497-503.

23. Di Rocco, F.; Parisi, G.; Zambelli, A.; Vida-Rioja, L. Rapid Evolution of Cytochrome c Oxidase Subunit II in Camelids (Tylopoda, Camelidae). J Bioenerg Biomembr 2006, 38, 293-297.

24. da Fonseca, R.R.; Johnson, W.E.; O’Brien, S.J.; Ramos, M.J.; Antunes, A. The Adaptive Evolution of the Mammalian Mitochondrial Genome. BMC Genomics 2008, 9, 119.

25. Da Silva, C.C.; Tomasco, I.H.; Hoffmann, F.G.; Lessa, E.P. Genes and Ecology: Accelerated Rates of Replacement Substitutions in the Cytochrome b Gene of Subterranean Rodents. The Open Evolution Journal 2009, 3.

26. Bondareva, O.V.; Potapova, N.A.; Konovalov, K.A.; Petrova, T.V.; Abramson, N.I. Searching for Signatures of Positive Selection in Cytochrome b Gene Associated with Subterranean Lifestyle in Fast-Evolving Arvicolines (Arvicolinae, Cricetidae, Rodentia). BMC Ecol Evol 2021, 21, 1-12.

27. Abramson, N.I.; Golenishchev, F.N.; Bodrov, S.Y.; Bondareva, O.V.; Genelt-Yanovskiy, E.A.; Petrova, T.V. Phylogenetic Relationships and Taxonomic Position of Genus Hyperacrius (Rodentia: Arvicolinae) from Kashmir Based on Evidences from Analysis of Mitochondrial Genome and Study of Skull Morphology. PeerJ 2020, 8, e10364.

28. Abramson, N.I.; Bodrov, S.Y.; Bondareva, O.V.; Genelt-Yanovskiy, E.A.; Petrova, T.V. Mitochondrial Genome Phylogeny of Voles and Lemmings (Rodentia: Arvicolinae): Evolutionary and Taxonomic Implications. bioRxiv 2021.

29. Bondareva, O.V.; Abramson, N.I. The Complete Mitochondrial Genome of the Common Pine Vole Terricola Subterraneus (Arvicolinae, Rodentia). Mitochondrial DNA Part B 2019, 4, 3925-3926.

30. Bondareva, O.V.; Mahmoudi, A.; Bodrov, S.Y.; Genelt-Yanovskiy, E.A.; Petrova, T.V.; Abramson, N.I. The Complete Mitochondrial Genomes of Three Ellobius Mole Vole Species (Rodentia: Arvicolinae). Mitochondrial DNA B: Resour 2020, 5, 2485-2487.

31. Cong, H.; Kong, L.; Liu, Z.; Wu, Y.; Motokawa, M.; Harada, M.; Li, Y. Complete Mitochondrial Genome of the Mandarin Vole Lasiopodomys Mandarinus (Rodentia: Cricetidae). Mitochondrial DNA A: Resour 2016, 27, 760-761.

32. Fedorov, V.B.; Goropashnaya, A.V. Complete Mitochondrial Genomes of the North American Collared Lemmings Dicrostonyx Groenlandicus Traill, 1823 and Dicrostonyx Hudsonius Pallas, 1778 (Rodentia: Arvicolinae). Mitochondrial DNA B: Resour 2016, $1,878-879$.

33. Fedorov, V.B.; Goropashnaya, A.V. Complete Mitochondrial Genome of the Eurasian Collared Lemming Dicrostonyx Torquatus Pallas, 1779 (Rodentia: Arvicolinae). Mitochondrial DNA B: Resour 2016, 1, 824-825.

34. Folkertsma, R.; Westbury, M.V.; Eccard, J.A.; Hofreiter, M. The Complete Mitochondrial Genome of the Common Vole, Microtus Arvalis (Rodentia: Arvicolinae). Mitochondrial DNA B: Resour 2018, 3, 446-447.

35. Hao, H.; Liu, S.; Zhang, X.; Chen, W.; Song, Z.; Peng, H.; Liu, Y.; Yue, B. Complete Mitochondrial Genome of a New Vole Proedromys Liangshanensis (Rodentia: Cricetidae) and Phylogenetic Analysis with Related Species: Are There Implications for the Validity of the Genus Proedromys? Mitochondrial DNA 2011, 22, 28-34.

36. Kang, C.; Yue, H.; Liu, M.; Huang, T.; Liu, Y.; Zhang, X.; Yue, B.; Zeng, T.; Liu, S. The Complete Mitochondrial Genome of Cricetulus Kamensis (Rodentia: Cricetidae). Mitochondrial DNA A: Resour 2016, 27, 976-977.

37. Partridge, M.A.; Davidson, M.M.; Hei, T.K. The Complete Nucleotide Sequence of Chinese Hamster (Cricetulus Griseus) Mitochondrial DNA: Full Length Research Article. DNA Sequence 2007, 18, 341-346. 
38. Triant, D.A.; DeWoody, J.A. Accelerated Molecular Evolution in Microtus (Rodentia) as Assessed via Complete Mitochondrial Genome Sequences. Genetica 2006, 128, 95-108.

39. Zhang, Z.; Sun, T.; Kang, C.; Liu, Y.; Liu, S.; Yue, B.; Zeng, T. The Complete Mitochondrial Genome of Lesser Long-Tailed Hamster Cricetulus Longicaudatus (Milne-Edwards, 1867) and Phylogenetic Implications. Mitochondrial DNA A: Resour. 2016, 27, 1303-1304.

40. Zhao, H.; Qi, X.; Li, C. Complete Mitochondrial Genome of the Muskrat (Ondatra Zibethicus) and Its Unique Phylogenetic Position Estimated in Cricetidae. Mitochondrial DNA B: Resour. 2018, 3, 296-298.

41. Gromov, I.M.; Polyakov, I.Y. Fauna of the USSR. Mammals. Tome 3. Vyp. 8. Voles (Microtinae); Nauka: Leningrad, 1977; Vol. 3;.

42. Musser, G.G.; Carleton, M.D. Superfamily Muroidea. In Mammal species of the world: a taxonomic and geographic reference; JHU Press, 2005; pp. 894-1531.

43. Abramson, N.I.; Lissovsky, A.A. Subfamily Arvicolinae. In The mammals of Russia: A taxonomic and geographic reference; Pavlinov, I.Y., Lissovsky, A.A., Eds.; KMK Scientific Press, 2012; pp. 127-141.

44. Lessa, E.P.; Vassallo, A.I.; Verzi, D.H.; Mora, M.S. Evolution of Morphological Adaptations for Digging in Living and Extinct Ctenomyid and Octodontid Rodents. Biol J Linn Soc 2008, 95, 267-283.

45. Smorkatcheva, A.V.; Lukhtanov, V.A. Evolutionary Association between Subterranean Lifestyle and Female Sociality in Rodents. Mamm Biol 2014, 79, 101-109.

46. Shubin, I.G. The mole voles (Ellobius). In Mammals of Kazakhstan. Vol.1 Gerbils, voles, Altai zokor; Sludsky, A.A., Ed.; Publishing House Nauka of Kazakh SSR: Almaata, 1978; Vol. 1, pp. 188-207.

47. Gambaryan P.P. Adaptive Features of the Locomotory Organs in Fossorial Mammals; Izdatel'stvo AN Armyanskoi SSR: Erevan, 1960;

48. Salvioni, M. Home Range and Social Behavior of Three Species of European Pitymys (Mammalia, Rodentia). Behav Ecol Sociobiol 1988, 22, 203-210.

49. Kurta, A. Mammals of the Great Lakes Region; University of Michigan Press: Ann Arbor, 1995;

50. Giannoni, S.M.; Borghi, C.E.; Martínez-Rica, J.P. Comparing the Burrowing Behaviour of the Iberian Mole Voles (Microtus (Terricola) Lusitanicus, M. (T.) Pyrenaicus and M. (T.) Duodecimcostatus). Mammalia 1993, 57.

51. Krystufek, B.; Vohralik, V. Mammals of Turkey and Cyprus: Rodentia I: Sciuridae, Dipodidae, Gliridae, Arvicolinae; Zgodovinsko drustvo za juzno Primorsko, 2005;

52. Mironov, A.D. Spatial Organization of Common Pine Vole (Microtus Subterraneus Selys-Longchamps, 1836) Colonies. Amurian Zoological Journal 2020, 12, 460-476.

53. Smorkatcheva, A.V. The Social Organization of the Mandarine Vole, Lasiopodomys Mandarinus, during the Reproductive Period. Z Säugetierkd 1999, 64, 344-355.

54. Darling, A.C.E.; Mau, B.; Blattner, F.R.; Perna, N.T. Mauve: Multiple Alignment of Conserved Genomic Sequence With Rearrangements. Genome Res 2004, 14, 1394-1403.

55. Katoh, K.; Standley, D.M. MAFFT Multiple Sequence Alignment Software Version 7: Improvements in Performance and Usability. Mol Biol Evol 2013, 30, 772-780.

56. Ronquist, F.; Teslenko, M.; van der Mark, P.; Ayres, D.L.; Darling, A.; Höhna, S.; Larget, B.; Liu, L.; Suchard, M.A.; Huelsenbeck, J.P. MrBayes 3.2: Efficient Bayesian Phylogenetic Inference and Model Choice Across a Large Model Space. Syst Biol 2012, 61, 539-542.

57. Drummond, A.J.; Rambaut, A. BEAST: Bayesian Evolutionary Analysis by Sampling Trees. BMC Evol Biol 2007, 7, 214.

58. Hassanin, A.; Léger, N.; Deutsch, J. Evidence for Multiple Reversals of Asymmetric Mutational Constraints during the Evolution of the Mitochondrial Genome of Metazoa, and Consequences for Phylogenetic Inferences. Syst Biol 2005, 54, 277298.

59. Lobry, J.R. Properties of a General Model of DNA Evolution under No-Strand-Bias Conditions. J Mol Evol 1995, 40, 326-330. 
60. Cock, P.J.A.; Antao, T.; Chang, J.T.; Chapman, B.A.; Cox, C.J.; Dalke, A.; Friedberg, I.; Hamelryck, T.; Kauff, F.; Wilczynski, B.; et al. Biopython: Freely Available Python Tools for Computational Molecular Biology and Bioinformatics. Bioinformatics 2009, 25, 1422-1423.

61. R Core Team (2020). - European Environment Agency Available online: https://www.eea.europa.eu/data-andmaps/indicators/oxygen-consuming-substances-in-rivers/r-development-core-team-2006 (accessed on 11 February 2021).

62. Yuan, F.; Nguyen, H.; Graur, D. ProtParCon: A Framework for Processing Molecular Data and Identifying Parallel and Convergent Amino Acid Replacements. Genes 2019, 10, 181.

63. Yang, Z.; Nielsen, R.; Goldman, N.; Pedersen, A.-M.K. Codon-Substitution Models for Heterogeneous Selection Pressure at Amino Acid Sites. Genetics 2000, 155, 431-449.

64. Yang, Z. PAML 4: Phylogenetic Analysis by Maximum Likelihood. Mol Biol Evol 2007, 24, 1586-1591.

65. Smith, M.D.; Wertheim, J.O.; Weaver, S.; Murrell, B.; Scheffler, K.; Kosakovsky Pond, S.L. Less Is More: An Adaptive BranchSite Random Effects Model for Efficient Detection of Episodic Diversifying Selection. Mol Biol Evol 2015, 32, $1342-1353$.

66. Wertheim, J.O.; Murrell, B.; Smith, M.D.; Kosakovsky Pond, S.L.; Scheffler, K. RELAX: Detecting Relaxed Selection in a Phylogenetic Framework. Mol Biol Evol 2015, 32, 820-832.

67. Murrell, B.; Wertheim, J.O.; Moola, S.; Weighill, T.; Scheffler, K.; Kosakovsky Pond, S.L. Detecting Individual Sites Subject to Episodic Diversifying Selection. PLoS Genet 2012, 8, e1002764.

68. Delport, W.; Poon, A.F.Y.; Frost, S.D.W.; Kosakovsky Pond, S.L. Datamonkey 2010: A Suite of Phylogenetic Analysis Tools for Evolutionary Biology. Bioinformatics 2010, 26, 2455-2457.

69. Pond, S.L.K.; Muse, S.V. HyPhy: Hypothesis Testing Using Phylogenies. In Statistical Methods in Molecular Evolution; Statistics for Biology and Health; Springer-Verlag: New York, 2005; pp. 125-181.

70. Huerta-Cepas, J.; Serra, F.; Bork, P. ETE 3: Reconstruction, Analysis, and Visualization of Phylogenomic Data. Mol Biol Evol 2016, 33, 1635-1638.

71. Tomasco, I.H.; Lessa, E.P. The Evolution of Mitochondrial Genomes in Subterranean Caviomorph Rodents: Adaptation against a Background of Purifying Selection. Mol Phylogenet Evol 2011, 61, 64-70.

72. Tavares, W.C.; Seuánez, H.N. Changes in Selection Intensity on the Mitogenome of Subterranean and Fossorial Rodents Respective to Aboveground Species. Mamm Genome 2018, 29, 353-363.

73. Zhang, T.; Lin, G.; Nevo, E.; Yang, C.; Su, J. Cytochrome b Gene Selection of Subterranean Rodent Gansu Zokor Eospalax Cansus (Rodentia, Spalacidae). Zool Anz 2013, 252, 118-122.

74. Bromham, L.; Rambaut, A.; Harvey, P.H. Determinants of Rate Variation in Mammalian DNA Sequence Evolution. J Mol Evol 1996, 43, 610-621.

75. Martin, A.P. Metabolic Rate and Directional Nucleotide Substitution in Animal Mitochondrial DNA. Mol Biol Evol 1995, 12, 1124-1131.

76. Martin, A.P.; Palumbi, S.R. Body Size, Metabolic Rate, Generation Time, and the Molecular Clock. Proc Natl Acad Sci USA 1993, 90, 4087-4091.

77. Lopez, J.V.; Culver, M.; Stephens, J.C.; Johnson, W.E.; O’Brien, S.J. Rates of Nuclear and Cytoplasmic Mitochondrial DNA Sequence Divergence in Mammals. Mol Biol Evol 1997, 14, 277-286.

78. Abramson, N.I.; Lebedev, V.S.; Tesakov, A.S.; Bannikova, A.A. Supraspecies Relationships in the Subfamily Arvicolinae (Rodentia, Cricetidae): An Unexpected Result of Nuclear Gene Analysis. Mol Biol 2009, 43, 834.

79. Galewski, T.; Tilak, M.; Sanchez, S.; Chevret, P.; Paradis, E.; Douzery, E.J. The Evolutionary Radiation of Arvicolinae Rodents (Voles and Lemmings): Relative Contribution of Nuclear and Mitochondrial DNA Phylogenies. BMC Evol Biol 2006, 6, 80.

80. Steppan, S.J.; Schenk, J.J. Muroid Rodent Phylogenetics: 900-Species Tree Reveals Increasing Diversification Rates. PLOS ONE 2017, 12, e0183070. 
81. Vereshchagin, N.K. The mammals of the Caucasus; a history of the evolution of the fauna. (Mlekopitayushchie Kavkaza; istoriya formirovaniya fauny).; Publ. Acad. Nauk USSR: Jerusalem, 1959;

82. Vorontsov, N.N. New Data on the Biology and Taxonomic Position of the Long-Clawed Mole Vole (Prometheomys Schaposchnikovi Satunin, 1901). Zoologicheskii Zhurnal 1966, 45, 619-623.

83. Gambaryan, P.P.; Karapetyan, V.S.; Ayrumyan, K.A.; Kazaryan, K.G.; Mezhlumyan, S.K. To the Ecology of the Long-Clawed Mole Vole Prometheomys Schaposchnikovi Sat. Materials on the study of the fauna of the Armenian SSR (Zoologicheskii Sbornik, 10) 1957, 3, 5-16.

84. Zimina, R.P.; Yasny, E.V. Observations on the Ecology of the Long-Clawed Mole Vole. Zool Zhurnal 1977, 82, 24-30.

85. Smorkatcheva, A.V.; Aksenova, T.G.; Zorenko, T.A. Ecology of the Chinese Vole Lasiopodomys Mandarinus (Rodentia, Cricetidae) in Transbaikalia. Zoologicheskii Zhurnal 1990, 69, 115-124.

86. Sun, H.; Zhang, Y.; Wang, B.; Li, Y.; Xu, W.; Mao, R.; Wang, Z. Investigation on Oxygen and Carbon Dioxide Fluctuations in Lasiopodomys Mandarinus Burrows. Pak J Zool 2019, 51.

87. Aulagnier, S.; Haffner, P.; Mitchell-Jones, A.J.; Moutou, F.; Zima, J. Mammals of Europe, North Africa and the Middle East; Bloomsbury Wildlife, 2018.

88. Caroli, L.; Capizzi, D.; Luiselli, L. Reproductive Strategies and Life-History Traits of the Savi's Pine Vole, Microtus Savii. Zool Sci 2000, 17, 209-216.

89. Jemioło, B. Reproduction in a Laboratory Colony of the Female Pine Voles, Pitymys Subterraneus. Acta Theriol 1983, 28, 197207.

90. Schröpfer, R. Die Postnatale Entwicklung Der Kleinwühlmaus, Pitymys Subterraneus De Selys-Longchamps, 1836 (Rodentia, Cricetidae). Bonn Zool Beitr 1977, 28, 249-268. 